\title{
PLANT DIVERSITY IN HAZARIKHIL WILDLIFE SANCTUARY OF CHITTAGONG AND ITS CONSERVATION MANAGEMENT
}

\author{
Rahman, M. A. \\ Department of Botany, University of Chittagong, Chittagong 4331, Bangladesh
}

\begin{abstract}
Plant diversity inventory in the Hazarikhil Wildlife Sanctuary of Bangladesh has been made through qualitative survey and their status of occurrence assessed for taking proper conservation management. The inventory has recognized 89 dicot (Magnoliopsida) families represented in this wildlife sanctuary by 305 genera and 478 species with in an area of 2908.5 ha. Of these, $86(18 \%)$ species were assessed to be rare and endangered while 14 (2.93\%) critically endangered. Determination of habit type resulted $189(39.54 \%)$ species of trees, $119(24.90 \%)$ shrubs including $26(5.44 \%)$ climbers and $170(35.56 \%)$ herbs including 46 climbers and two epiphytes. Three species, thought to be possibly extinct from the flora of Bangladesh, are being reported as rediscovery from this sanctuary, these are: Aglaia edulis (Roxb.) Wall., Knema erratica (Hook.f. and Thom.) J. Sinclair and Artabotrys caudatus Wall ex Hook.f. and Thom. An enumeration of these taxa is prepared citing each species with family name, bangla/local name(s), habit and conservation status for supporting the preparation of protected area management plan for implementation of National Conservation Strategy.
\end{abstract}

Key words: Plant diversity, inventory, assessment, conservation status, Hazarikhil wildlife sanctuary.

\section{INTRODUCTION}

The Hazarikhil reserve forest of Chittagong is one of the richest biodiversity resource areas of Bangladesh and has been declared as wildlife sanctuary for taking proper protection and conservation management. It occupies an area of 2908.5 ha and lies between $22^{\circ}$ $40^{\prime}-22^{\circ} 46^{\prime} \mathrm{N}$ latitude and $91^{\circ} 38^{\prime}-91^{\circ} 42^{\prime} \mathrm{E}$ longitude, located to Fatikchari Upazila of Chittagong district, under Ramgarh-Sitakundu Reserve Forest-2 of Chittagong North Forest Division. It includes 3 forest blocks under Hazarikhil Forest Range: Hazarikhil (950.20 ha) and Rangapani (621.05 ha) of Hazarikhil forest beat and Harowalchari (1337.25 ha) of Fatikchari forest beat. The topography of Hazarikhil forests constituted with high (152 to $365 \mathrm{~m}$ ), medium (76 to $152 \mathrm{~m}$ ) and low hills with 15 to $76 \mathrm{~m}$ altitude. These hills are made up of sand-storms and shale.

The forest of Hazarikhil is a part of the flora of Ramgarh - Bandarban hills which is floristically and geographically more related to Indo-China flora (Khan 1977). The floristic composition of Hazarikhil hills like other vegetations of Chittagong is similar or more related to that of the vegetation of tropical Myanmar.

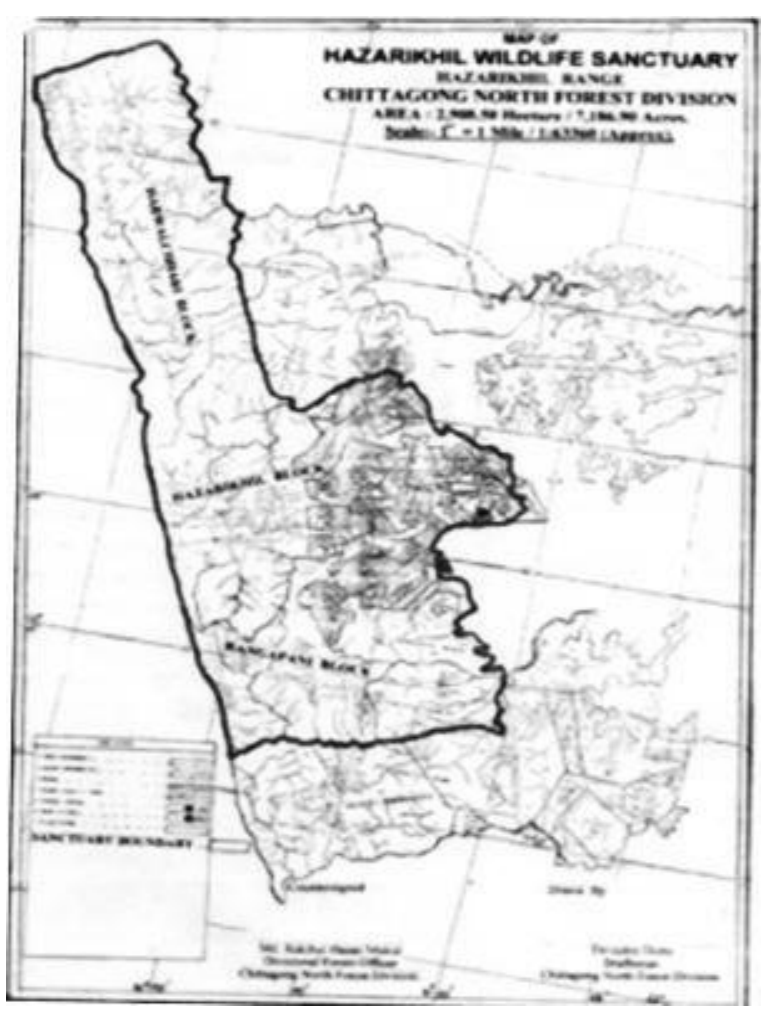

Fig. 1. Map of Hazarikhil wildlife sanctuary.

The flora of this wildlife sanctuary is mostly evergreen and mixed evergreen type except a few blocks of deciduous type composed of Teak plantations. Natural vegetation mostly with forest trees are on the discontinuous hills of low, medium and high elevations. Artificial plantations start in this forest 
since 1921 and significant natural forest areas have been converted to teak and recent acacia plantations. The present investigation was envisaged to assess plant diversity in the Hazarikhil Wildlife Sanctuary aimed at justifying the declaration of WS in this natural forest.

\section{MATERIAL AND METHODS}

The survey of the flora has been made through repeated field visits. Listing of known species and collection of specimens with field data for identification and preservation in Taxonomic Data Bank (TDB) have been made. Collections of fertile specimens have been made for identification and preservation as voucher specimens. Data on the status of occurrence, abundance, threats and conservation status, local names, where ever available, were recorded, so far, for individual species based on qualitative survey and investigation throughout the flora.

Identification of unknown species has been made in the Herbarium of Chittagong University (HCU) consulting specimens/taxonomists of Bangladesh Forest Research Institute Herbarium (BFRIH) and Bangladesh National Herbarium (DACB). Nomenclatural determination with current names/synonyms has been made consulting relevant literature, such as, Ahmed et al. (2007, 2008, 2009), Rashid and Rahman (2011, 2012), Rahman (2013), Kew Plant List (internet) and different Floras of neighboring countries, like, India, Bhutan, Nepal, Myanmar etc. Local/Bangla names of many plants have been collected from local people during field trips and by consulting Prain (1903), Heinig (1926), Huq (1986), Das and Alam (2001), Dey (1995) and Dey et al. (1999).

The recognized taxa of this WS are enumerated in Table 1, citing each species with family name, bangle/local name(s), habit, potential value and status of occurrence. The species are arranged family wise in alphabetical order. The families are also arranged alphabetically.

\section{RESULTS AND DISCUSSION}

The flora of Hazarikhil Wildlife Sanctuary is composed of 189 trees, 119 shrubs and 170 herbs (Table 1) within an area of 2908.5 ha. The study revealed that the sanctuary is composed of a number of small and fragmented natural forests with rich species diversity and some patches of planted forests with gorjan, chapalish, teak, mehagini, gamar, telsure, chikrassi, dumur, jam, bhadi, bokain, udhal, mango, rubber etc. The palms, rattans and bamboos mostly occupy the valleys. Common shrubs, herbs, grasses and babanas are fragmented to degraded habitats. A few individuals of Boilam, Civit and Lohakat are still available as characteristic elements of the forest. Number of the species of edible fruits and wild fruit trees are not sufficient for wildlife support. Among fruit trees, amlaki, haritaki, Kawgola, painnagola, lotkan, gab, dumur, barta, buttijam, phuljam etc. are seavailable. Among epiphytes, species of Cymbidium, Arides, Dendrobium, Drynaria, Raphidophra, Phothos etc. are more or less dominant. Natural habitats like waterfall and stony charas (e.g. Harowalchari chara), although under degradation, are still support luxuriant growth of aroids, begonias, arecas, palms, ferns and bryophytes.

Table 1. Plants of Hazarikhil Wildlife Sanctuary of Chittagong.

\begin{tabular}{|c|c|c|c|c|}
\hline Family & Species & Bangla/local name & Habit & $\begin{array}{l}\text { Status of } \\
\text { occurance }\end{array}$ \\
\hline \multirow{6}{*}{ Acanthaceae } & Andrographis laxiflora (Blume) Lindau. & - & Herb & Common \\
\hline & A. paniculata (Burm. f.) Wall. ex Nees & Kalomegh & Herb & Common \\
\hline & E. paltiferum (Wall.) Nees & - & Shrub & Common \\
\hline & Hygrophila polysperma (Roxb.) T. Anders & - & Herb & Common \\
\hline & J. gendarussa L. & Jagat mardan, Nilnishinda & Shrub & Common \\
\hline & Lapithogathis incurva Buch.-Ham. & - & Herb & Common \\
\hline
\end{tabular}




\begin{tabular}{|c|c|c|c|c|}
\hline & Nelsonia canescens (Lam.) Spreng. & Paramul & Herb & Common \\
\hline & Ruellia acuminata $\mathrm{L}$. & - & Herb & Common \\
\hline & R. tuberosa L. & Chatpoti & Herb & Common \\
\hline & Rungia pectinata (L.) Nees & Birlongopark Punaka pundu & Herb & Common \\
\hline & Staurogyne argentea Wall. & - & Herb & Common \\
\hline & S. angustifolia $\mathrm{T}$. Anders. & -- & Herb & Common \\
\hline & Strobilanthes auriculatus Nees & Kara, & Shrub & Rare \\
\hline & S. scaber Nees & - & Herb & Rare \\
\hline & Thunbergia alata Bojer ex Sims & - & Herbaceous climber & Rare \\
\hline & T. grandiflora Roxb. & - & Herbaceous climber & Common \\
\hline Actinidiaceae & Saurauia roxburghii Wall. & Dalup & Tree (small) & Rare \\
\hline \multirow{8}{*}{ Amaranthaccae } & Cyathula prostate Blume & - & Herb & Common \\
\hline & Achyranthes aspera $\mathrm{L}$. & Apang & Herb & Common \\
\hline & Alternanthera sessilis & Chanchi & Herb & Common \\
\hline & A. philoxeroides (Mart.) Griseb. & Helencha & Herb & Common \\
\hline & Amaranthus spinosus L. & Kantanotey, Katamaris & Herb & Common \\
\hline & A. viridis $\mathrm{L}$. & Notey, Nateyshak, Maris sag & Herb & Common \\
\hline & Celosia argentea $\mathrm{L}$. & Moragh phul & Herb & Common \\
\hline & Gomphrena celosioides Mart. & - & Herb & Common \\
\hline \multirow{10}{*}{ Anacardiaceae } & Bouea oppositifolia (Roxb.) Maessn. & Mailam, Uriam, Miriam & Tree (medium) & $\begin{array}{l}\text { Critically } \\
\text { Endangered }\end{array}$ \\
\hline & Buchanania lancifolia Roxb. & Chikki & Tree & Rare \\
\hline & Drimycarpus racemosus Hook.f. & Kodi-barele, Nala-amshi & Tree & $\begin{array}{l}\text { Critically } \\
\text { Endangered }\end{array}$ \\
\hline & Holigarna longifolia Roxb. & Barola, Rakta-Barola & Tree & $\begin{array}{l}\text { Critically } \\
\text { Endangered }\end{array}$ \\
\hline & Lannea coromandelica (Houtt.) Merr. & Jialbhadi, Jiga, Kapila, Bhadi & Tree & Common \\
\hline & Mangifera indica $\mathrm{L}$. & Aam & Tree & Planted \\
\hline & M. longipes Griff. & Jangali Aam & Tree & $\begin{array}{l}\text { Critically } \\
\text { Endangered }\end{array}$ \\
\hline & M. sylvatica Roxb. ex Wall. & $\begin{array}{l}\text { Uri Aam, Jangali Aam, } \\
\text { Laksmi Aam, Kush um, }\end{array}$ & Tree & $\begin{array}{l}\text { Critically } \\
\text { Endangered }\end{array}$ \\
\hline & Spondias pinnata (L. f.) Kurz & Amra, Deshi-Amra, Pial & Tree & Rare \\
\hline & Swintonia floribunda Griff. & Civit, Chundul, Am chundul & Ttree & $\begin{array}{l}\text { Critically } \\
\text { Endangered }\end{array}$ \\
\hline \multirow{8}{*}{ Annonaceae } & Artabotrys caudatus Wall & Not available & Woody climber & Endangered \\
\hline & Desmos dumosus (Roxb.) Saff. & - & Shrubby climber & Endangered \\
\hline & Fissistigma rubiginosum (A. DC.) Merr. & - & Shrubby climber & Endangered \\
\hline & F. wallichii (Hook.f. \& Thom.) Merr. & - & Woody climber & Endangered \\
\hline & Miliosa globosa G. Panigr. \& Mishra & Not available & Tree & $\begin{array}{l}\text { Critically } \\
\text { Endangered }\end{array}$ \\
\hline & $\begin{array}{l}\text { M. longiflora (Hook.f. \& Thom.) Finet } \\
\& \quad \text { Gagnep. }\end{array}$ & $\begin{array}{l}\text { Phesicow,Roktagola } \\
\text { Horinagola }\end{array}$ & Tree & $\begin{array}{l}\text { Critically } \\
\text { Endangered }\end{array}$ \\
\hline & Uvaria cordata (Dunal) Alston & - & Woody climber & Endangered \\
\hline & U. hamiltonii Hook.f. \& Thom. & Latkan & Woody climber & Endangered \\
\hline \multirow[t]{2}{*}{ Apiaceae } & Centella asiatica $(\mathrm{L}$.$) Urban$ & $\begin{array}{l}\text { Thankuni, Ada gongoni, Ada } \\
\text { molki, Adamoni }\end{array}$ & Herbaceous trailer & Common \\
\hline & Hydrocotyle sibthorpioides Lam. & - & Herbaceous trailer & Common \\
\hline \multirow{8}{*}{ Apocynaceae } & Alstonia nerifolia & Chatim & Tree & Rare \\
\hline & Alstonia scholaris (L.) R. Br. & Chatim & Tree & Common \\
\hline & Anodendron manubriatum (Wall.) Merr. & Dul & Woody climber & $\begin{array}{l}\text { Rare/ } \\
\text { Endanoered }\end{array}$ \\
\hline & Chonemorpha fragrans (Moon) Alston & Gar badero & Woody climber & Endangered \\
\hline & Holarhena antidysenterica (L.) Wall. ex DC. & Kurchi & Tree (small) & Common \\
\hline & Ichnocarpus frutescens (L.) R. Br. & Dudhilata & Woody twiner & Common \\
\hline & Melodinus monogynus Roxb. & - & Woody twiner & Endangered \\
\hline & Rauvolfia serpentina (L.) Benth. ex Kurz & Sarpagandha & Herb & Endangered \\
\hline
\end{tabular}




\begin{tabular}{|c|c|c|c|c|}
\hline & Strophanthus wallichii Decne & - & Shrubby climber & Endangered \\
\hline & Tabernaemontana recurva Roxb. & Choota katwadar, Rupa tola & Shrub & Rare \\
\hline & Vallaris solanacea (Roxb.) Kuntze & Hadpur, Mali & Scandent Shrub & $\begin{array}{l}\text { Critically } \\
\text { Endangered }\end{array}$ \\
\hline & Wrightia arborea (Dennst.) Mabb. & Dudh-koraiya & Tree & Common \\
\hline \multirow{2}{*}{ Araliaceae } & Brassaiopsis glomerulata (Blume) Regel & Kurila & Tree (small) & Common \\
\hline & Trevesia palmata (Roxb.) Vis. & Vombal, Kawhtebel & Tree (small) & Common \\
\hline \multirow{2}{*}{ Aristolochiaceae } & Aristolochia tagala Cham. & Ishwarmul & Herbaceous climber & Common \\
\hline & Aristolochia indica $\mathrm{L}$. & Ishwarmul & Herbaceous climber & Rare \\
\hline \multirow{8}{*}{ Asclepiadaceae } & Asclepias curassavica $\mathrm{L}$. & Moricha & Herb & Rare \\
\hline & Calotropis gigantea (L.) R. Br. & Akanda & Shrub & Common \\
\hline & Gymnema acuminatum (Roxb.) Wall. & Khara lata & Woody twiner & Rare \\
\hline & Hemidesmus indicus (L.) R. Br. & Anantamul & Herb & Endangered \\
\hline & Hoya globolosa Hook, f. & Pargacha & Herbaceous Epiphyte & Rare \\
\hline & Hoya globusa Hook.f. & & Herbaceous Epiphyte & Endangered \\
\hline & Hoya parasitica (Roxb) Wall. ex Wight & Pargacha & Herbaceous Epiphyte & Rare \\
\hline & Tylophora indica (Burm. F.) Merr. & Antamul, Anantamul & Herbaceous twiner & Rare \\
\hline \multirow{22}{*}{ Asteraceae } & Adenostemma lavenia (L.) Kuntze & Baro-kesuti & Herb & Common \\
\hline & Ageratum conyzoides $\mathrm{L}$. & Fulkuri & Herb & Common \\
\hline & Blumea fistulosa (Roxb.) Kurz & - & Herb & Common \\
\hline & B. lacera (Brum. F.) DC. & Kukursunga, Barakukshima & Herb & Common \\
\hline & B. lanceolaria (Roxb.) Druce & - & Herb & Common \\
\hline & Blumeopsis flava (DC.) Gagnep & - & Herb & Common \\
\hline & Chromolaena odorata (L.) & Barashialmati, Assamlata & Herb & Common \\
\hline & Crassocephalum crepidioides S. Moore & - & Herb & Common \\
\hline & Eclipta alba (L.) Hassk. & Kesoraj, Kalokeshi, Keshute & Herb & Common \\
\hline & Elephantopus scaber L. & Shamdalan, Gajiashak & Herb & Common \\
\hline & Emilia sonchifolia (L.) DC. & Mechitra, Sadimudi & Herb & Common \\
\hline & Mikania cordata (Burm. F.) Robinson & $\begin{array}{l}\text { Tufanilata, } \\
\text { Shankunilata }\end{array}$ & Herbaceous climber & Common \\
\hline & Pseudelephantopus spicatus Rohr & - & Herb & Common \\
\hline & Sonchus wightianus DC. & - & Herb & Common \\
\hline & Sphaeranthus africanus L. & - & Herb & Common \\
\hline & Sphaeranthus indicus L. & Murmuri & Herb & Common \\
\hline & Sphaeranthus senegalensis DC. & - & Herb & Common \\
\hline & Spilanthes calva DC. & Marhata-tiga & Herb & Common \\
\hline & Syndrella nodiflora (L.) Gaertn. & - & Herb & Common \\
\hline & Tridax procumbens $\mathrm{L}$. & Tridhara & Herb & Common \\
\hline & Vernonia cinerea (L.) Less. & Kukshim, Sahadebi, Shillata & Herb & Common \\
\hline & Xanthium indicum Koenig & Lehra, Ghagra & Herb & Common \\
\hline \multirow{2}{*}{ Begoniaceae } & Begonia sp. & - & Herb (succulent) & Endangered \\
\hline & Begonia roxburghii (Miq.) A. DC. & - & Herb (succulent) & Endangered \\
\hline Betulaceae & Alnus nepalensis G. Don & - & Tree & Endangered \\
\hline \multirow{2}{*}{ Bignoniaceae } & Oroxylum indicum (L.) Kurz & Thona & Tree & Rare \\
\hline & Stereospermum colais Mabberley & Dharmara & Tree & Rare \\
\hline Bixaceae & Bixa orellana $\mathrm{L}$. & Latkon, Harpara & Tree (small) & Rare \\
\hline \multirow{2}{*}{ Bombacaceae } & Bombax ceiba $\mathrm{L}$. & Simul, Tula & Tree & $\begin{array}{l}\text { Rare/ } \\
\text { planted }\end{array}$ \\
\hline & Bombax insigne Wall. & Ban-simul, Pahari-simul & Tree (large) & Rare \\
\hline Boraginaceae & Helitropium indicun $\mathrm{L}$ & Hatisur & Herb & Common \\
\hline Buddlejaceae & Buddleja asiatica Lour. & Neemda, Badhota & Shrub & Common \\
\hline Burseraceae & Protium serratum (Wall. ex Colebr.) Engl. & Gutgutya, Haliabhadi & Tree & Rare \\
\hline \multirow{4}{*}{ Caesalpinaccae } & Caesalpinia digyna Rottl. & Umul kuchi & Scandent Shrub & Common \\
\hline & Caesalpinia bonduc (L.) Roxb. & Nata, Natakaranga & Scandent Shrub & Rare \\
\hline & Cassia fistula $\mathrm{L}$. & Sonalu, Bandar-lati & Tree & Planted \\
\hline & Cassia javanica L. ssp. nodosa & Bander-lutia, Bon-sonalu & Tree & Rare \\
\hline
\end{tabular}




\begin{tabular}{|c|c|c|c|c|}
\hline & $\begin{array}{l}\text { Cassia obtusifolia } \mathrm{L} . \\
\text { C. siamea Lam. } \\
\text { C. sophera } \mathrm{L} . \\
\text { Senna alata }(\mathrm{L} .) \text { Roxb. } \\
\text { Saraca asoca }(\text { Roxb.) de Wilde. } \\
\text { Senna occidentalis (L.) Link } \\
\text { S.tora }(\text { L.) Roxb. } \\
\end{array}$ & $\begin{array}{l}\text { Chakunda } \\
\text { Minjiri, Minjuri } \\
- \\
\text { Dadmordan } \\
\text { Ashok } \\
\text { Bara-Kalkasunda, Eski } \\
\text { Teraj } \\
\end{array}$ & $\begin{array}{l}\text { Undershrub } \\
\text { Tree } \\
\text { Shrub } \\
\text { Shrub } \\
\text { Tree } \\
\text { Herb } \\
\text { Undershrub } \\
\end{array}$ & $\begin{array}{l}\text { Common } \\
\text { Planted } \\
\text { Common } \\
\text { Common } \\
\text { Rare } \\
\text { Common } \\
\text { Common } \\
\end{array}$ \\
\hline Campanulaceae & Campanumoea lancifolia (Roxb.) Merr. & - & Undershrub & Rare \\
\hline Capparaceae & $\begin{array}{l}\text { Cleome rutidosperma DC. } \\
\text { Cleome viscosa L. } \\
\text { Crateva manga (Lour.) DC. }\end{array}$ & $\begin{array}{l}- \\
\text { Halde hurhure } \\
\text { Barun, Gota baruna }\end{array}$ & $\begin{array}{l}\text { Herb } \\
\text { Herb } \\
\text { Tree (small) }\end{array}$ & $\begin{array}{l}\text { Common } \\
\text { Common } \\
\text { Common }\end{array}$ \\
\hline Casuarinaceae & Casuarina equisetifolia L. & Bilati jhau & Tree & Planted \\
\hline Celastraceae & Euоnyтиs attenuatus Wall. ex Laws. & - & Shrub & Rare \\
\hline Chenopodiaceae & Chenopodium album L. & Betushak, Vathua-sak & Herb & Common \\
\hline Clusiaceae & $\begin{array}{l}\text { Garcinia cowa Roxb. } \\
\text { Mesua floribunda (Wall.) Kosterm }\end{array}$ & $\begin{array}{l}\text { Kao-gola, Kawa, Kaglichu } \\
\text {-- }\end{array}$ & $\begin{array}{l}\text { Tree (medium) } \\
\text { Tree (large) }\end{array}$ & $\begin{array}{l}\text { Rare/Endan } \\
\text { Planted }\end{array}$ \\
\hline Combretaceae & $\begin{array}{l}\text { Calycopteris floribunda (Roxb.) Lam. } \\
\text { Combretum decandram Roxb. } \\
\text { Terminalia attenua (Gaertn) Roxb. } \\
\text { Terminalia alata Heyne ex Roth. } \\
\text { T. bellerica (Gaertn.) Roxb. } \\
\text { T. chebula Retz. }\end{array}$ & $\begin{array}{l}\text { Guicha lata } \\
\text { Kali gumuchi, Sada guicha } \\
\text { Hatiyal, Haritaki } \\
\text { Asal,Asna, Hasna, Saj } \\
\text { Bohera, Boyra } \\
\text { Haritaki, Gol horitaki }\end{array}$ & $\begin{array}{l}\text { Scandent Shrub } \\
\text { Scandent Shrub } \\
\text { Tree (large) } \\
\text { Tree (large) } \\
\text { Tree } \\
\text { Tree (large) }\end{array}$ & $\begin{array}{l}\text { Rare } \\
\text { Rare } \\
\text { Planted } \\
\text { Rare } \\
\text { Planted } \\
\text { Planted }\end{array}$ \\
\hline Connaraceae & Connarus paniculatus Roxb. & Katgular & Woody climber & Rare \\
\hline Convolvulaceae & $\begin{array}{l}\text { Argyria argentea (Roxb.) Chosy. } \\
\text { A. capitiformis (Poir.) van Cheek. Oostr. } \\
\text { Calystegia hederacea Wall. } \\
\text { Hewittia sublobata (L. f.) Kuntze } \\
\text { Ipomoea attenua Forsk. } \\
\text { I. alba } \mathrm{L} \\
\text { I. batatas (L.) Lam. } \\
\text { I. fistulosa Mart. ex Choisy } \\
\text { I. hederifolia } \text { L. } \\
\text { I. pes-tigridis L. } \\
\text { I. pileata Roxb. } \\
\text { Merremia attenuate (L.) Halleer } \\
\text { M. umbellata (L.) Halleer } \\
\text { Operculina turpethum (L.) S. Marso. }\end{array}$ & $\begin{array}{l}\text { Bara rupatola, Chotto-biztarak } \\
- \\
- \\
- \\
\text { Kalmi shak } \\
\text { Hal kalmi, Dudh kalmi } \\
\text { Misti Aloo } \\
\text { Dhol kalmi, Police lata } \\
\text { - } \\
\text { Languli lata } \\
\text { - } \\
\text { Sada kalmi } \\
\text { Sadakamni, Korma lata } \\
\text { Dudh Kalmi }\end{array}$ & $\begin{array}{l}\text { Herbaceous climber } \\
\text { Herbaceous climber } \\
\text { Herbaceous twiner } \\
\text { Herbaceous climber } \\
\text { Herbaceous trailer } \\
\text { Herbaceous climber } \\
\text { Herbaceous trailer } \\
\text { Shrub } \\
\text { Herbaceous climber } \\
\text { Herbaceous climber } \\
\text { Herbaceous climber } \\
\text { Herbaceous twiner } \\
\text { Herbaceous climber } \\
\text { Herbaceous climber }\end{array}$ & $\begin{array}{l}\text { Common } \\
\text { Common } \\
\text { Common } \\
\text { Common } \\
\text { Common } \\
\text { Common } \\
\text { Common } \\
\text { Common } \\
\text { Common } \\
\text { Common } \\
\text { Common } \\
\text { Common } \\
\text { Common } \\
\text { Common }\end{array}$ \\
\hline Cucurbitaceae & $\begin{array}{l}\text { Citrullus colocynthis (L.) Schrad. } \\
\text { Coccinia grandis (L.) Voigth } \\
\text { Hodgsonia macrocarpa (Blume) Cogn. }\end{array}$ & $\begin{array}{l}\text { Indrayan } \\
\text { Kawajhinga, Telakucha, Kuchila } \\
\text { Gular, Pathligular }\end{array}$ & $\begin{array}{l}\text { Herbaceous climber } \\
\text { Herbaceous climber } \\
\text { Herbaceous climber }\end{array}$ & $\begin{array}{l}\text { Endangered } \\
\text { Common } \\
\text { Critically } \\
\text { Endangered }\end{array}$ \\
\hline & $\begin{array}{l}\text { Thladiantha cordifolia (Blume) Cogn. } \\
\text { Trichosanthes cordata Roxb. } \\
\text { T. tricuspidata Lour. }\end{array}$ & $\begin{array}{l}\text { - } \\
\text { Bhuikakra } \\
\text { Makal }\end{array}$ & $\begin{array}{l}\text { Herbaceous climber } \\
\text { Herbaceous climber } \\
\text { Herbaceous climber }\end{array}$ & $\begin{array}{l}\text { Rare } \\
\text { Rare } \\
\text { Rare/Endan }\end{array}$ \\
\hline Cuscutaceae & Cuscuta reflexa Roxb. & Sorna lata, Jarbuti & Herbaceous climber & Common \\
\hline Datiscaceae & Tetrameles nodiflora $\mathrm{R} . \mathrm{Br}$. & Chundul, Maynakat & Tree (large) & Rare/Endan \\
\hline Dilleniaceae & $\begin{array}{l}\text { Dillenia pentagyna Roxb. } \\
\text { D. indica } \mathrm{L} \text {. }\end{array}$ & Hargaza & Tree & $\begin{array}{l}\text { Critically } \\
\text { Endangered } \\
\text { Planted }\end{array}$ \\
\hline Dipterocarpaceae & $\begin{array}{l}\text { Anisoptera scaphula (Roxb.) Pierre. } \\
\text { Dipterocarpus turbinatus Gaertn. } \\
\text { D. alatus Roxb. ex G. Don } \\
\text { D. costatus } \\
\text { Hopea odorata Roxb. }\end{array}$ & $\begin{array}{l}\text { Boilsur, Sada boilam } \\
\text { Kali garjan, Teli garjan, } \\
\text { Kuroli } \\
\text { Dholi garjan, Sil garjan } \\
\text { Garjan } \\
\text { Telsur }\end{array}$ & $\begin{array}{l}\text { Tree } \\
\text { Tree } \\
\text { Tree } \\
\text { Tree (large) }\end{array}$ & $\begin{array}{l}\text { Critically } \\
\text { Endangered } \\
\text { Rare/ } \\
\text { Planted } \\
\text { Rare/Cult } \\
\text { Rare } \\
\text { Rare/ Planted }\end{array}$ \\
\hline
\end{tabular}




\begin{tabular}{|c|c|c|c|c|}
\hline Droceraceae & Drosera burmannii Vahl & Surjasishir & Herb & $\begin{array}{l}\text { Critically } \\
\text { Endangered }\end{array}$ \\
\hline Ebenaceae & $\begin{array}{l}\text { Diospyros malabarica (Desr.) Koste. } \\
\text { D. racemosa Roxb. }\end{array}$ & $\begin{array}{l}\text { Gab, Deshi gab } \\
\text { Gab }\end{array}$ & $\begin{array}{l}\text { Tree (medium) } \\
\text { Tree (medium) }\end{array}$ & $\begin{array}{l}\text { Rare } \\
\text { Rare/Endan }\end{array}$ \\
\hline Elaeocarpaceae & $\begin{array}{l}\text { Elaeocarpus floribundus Blume } \\
\text { E. tectorius (Lour.) Poir. }\end{array}$ & $\begin{array}{l}\text { Jalpai, Belphoi } \\
\text { Chekio, Jalpai }\end{array}$ & $\begin{array}{l}\text { Tree (large) } \\
\text { Tree }\end{array}$ & $\begin{array}{l}\text { Planted } \\
\text { Rare }\end{array}$ \\
\hline Euphorbiaceae & $\begin{array}{l}\text { Antidesma acidum Retz. } \\
\text { A. bunius (L.) Spreng. } \\
\text { A. ghaesembilla Gaertn. } \\
\text { A. roxburghii Wall. ex Tul. } \\
\text { Aporosa wallichii Hook.f. } \\
\text { A. aurea Hook.f. } \\
\text { A. octandra A.R. Vickery. } \\
\text { Baccaurea ramiflora Lour. } \\
\text { Bischofia javanica Blume } \\
\text { Breynia retusa (Dennst.) Alston. } \\
\text { Briedelia stipularis (L.) Blume } \\
\text { B. tomentosa } \text { Blume } \\
\text { Chaetocarpus castanicarpa (Roxb.)Thw. } \\
\text { Chrozophora rottleri (Geisel.) A. Juss. } \\
\text { Cnesmone javanica Blume } \\
\text { Croton bonplandianus Bail } \\
\text { C. caudatus Geisel. } \\
\text { C. lobetus L. } \\
\text { Euphorbia hirta } \text { L. } \\
\text { Glochidion assamicum (Muell-Arg.) Hook.f. } \\
\text { G. multiloculare (Roxb. ex Willd.) Muell.- Arg. } \\
\text { G. spherogynum Kurz } \\
\text { G. lanciolarium (Roxb.) Voigt. } \\
\text { Macaranga denticulata (Blume) Muell.-Arg. } \\
\text { M. peltata (Roxb) Muell.-Arg. } \\
\text { Mallotus philippensis (Lam.) Muell.-Arg. } \\
\text { M. roxburghianus Muell.-Arg. } \\
\text { M. tetracoccus (Roxb.) Kurz } \\
\text { Phyllanthus attenuatus Poir } \\
\text { P. emblica L. } \\
\text { P. niruri L. } \\
\text { P. sikkimensis Muell.-Arg. } \\
\text { Sapium indicum Willd } \\
\text { S. baccatum Roxb. } \\
\text { Suregada multiflora (A. Juss.) Baill. } \\
\text { Tragia allidrate L. } \\
\text { T. nudiflora L. }\end{array}$ & $\begin{array}{l}\text { Multa, Khudi jam, Limtoa } \\
\text { Banshial buka } \\
\text { Khudijam } \\
\text { - } \\
\text { Karullah, Kakra, Kechua } \\
\text { Kechuan } \\
\text { Patha-kharullah, Ataligula } \\
\text { Latka, Latkon, Harpata, Vaccum } \\
\text { Kanjalbhadi } \\
\text { Silpati } \\
\text { Pat khowi } \\
\text { Khoi } \\
\text { Bul kakra } \\
\text { Khudi Okra } \\
\text { Chottra patha, Paharibichuti } \\
\text { Dongalas } \\
\text { Nanbhanti } \\
\text { - } \\
\text { Ghaopata, Dudhiya, Barakeru } \\
\text { - } \\
\text { Aniatori } \\
\text { - } \\
\text { Anguti, Bhauri, Kakra, Kechuan } \\
\text { Burna, Bura-kochi, Rata-bura } \\
\text { - } \\
\text { Kamalaguli } \\
\text { Nim puteli, Nunia kachi } \\
\text { Kumari-bura, Main bura } \\
\text { Panjuli } \\
\text { Amloki, Ambolothi, Aonla } \\
\text { Bhuiamla } \\
\text { - } \\
\text { Hura, Batul, Bara mel } \\
\text { Chotto mel, Katagola, Kala boil } \\
\text { Ban-naranga, Ban-naringa } \\
\text { Bichuti, Chitta } \\
\text { Pitali, Batal, Pitabara }\end{array}$ & $\begin{array}{l}\text { Large shrub } \\
\text { Tree (medium) } \\
\text { Tree (medium) } \\
\text { Tree (small) } \\
\text { Tree } \\
\text { Tree } \\
\text { Tree } \\
\text { Tree (medium) } \\
\text { Tree } \\
\text { Shrub } \\
\text { Tree (small) } \\
\text { Tree (small) } \\
\text { Tree (medium) } \\
\text { Herb } \\
\text { Shrubby climber } \\
\text { Herb } \\
\text { Shrub } \\
\text { Herb } \\
\text { Herb } \\
\text { Tree (small) } \\
\text { Tree (small) } \\
\text { Tree } \\
\text { Tree (small) } \\
\text { Tree (medium) } \\
\text { Tree } \\
\text { Tree (small) } \\
\text { Tree (small) } \\
\text { Tree (medium) } \\
\text { Shrub } \\
\text { Tree } \\
\text { Herb } \\
\text { Shrub } \\
\text { Tree (small) } \\
\text { Tree } \\
\text { Tree (medium) } \\
\text { Herb } \\
\text { Tree (medium) }\end{array}$ & $\begin{array}{l}\text { Rare } \\
\text { Rare } \\
\text { Rare } \\
\text { Rare } \\
\text { Rare } \\
\text { Rare } \\
\text { Rare } \\
\text { Rare } \\
\text { Common } \\
\text { Rare } \\
\text { Rare } \\
\text { Rare } \\
\text { Common } \\
\text { Common } \\
\text { Common } \\
\text { Common } \\
\text { Common } \\
\text { Common } \\
\text { Common } \\
\text { Rare } \\
\text { Rare } \\
\text { Rare } \\
\text { Rare } \\
\text { Common } \\
\text { Common } \\
\text { Rare } \\
\text { Rare } \\
\text { Rare } \\
\text { Rare } \\
\text { Planted } \\
\text { Common } \\
\text { Rare } \\
\text { Rare } \\
\text { Rare } \\
\text { Common } \\
\text { Common } \\
\text { Rare }\end{array}$ \\
\hline Fabaceae & $\begin{array}{l}\text { Abrus precatorius L. } \\
\text { A. pulchellus Wall. } \\
\text { Butea monosperma (Lam.) Kuntze } \\
\text { Crotalaria acicularis Buch.-Ham. ex Benth } \\
\text { C. tetragona Roxb. ex Anders. } \\
\text { C. pallid Aiton } \\
\text { C. dubia Graham ex Benth. } \\
\text { C. ferruginea } \text { Graham ex Benth. } \\
\text { C. verrucosa } \text { L. } \\
\text { Dalbergia sericea G. Don } \\
\text { D. lanceolaria } \text { L. f. } \\
\text { D. malabarica } \text { Prain } \\
\text { D. sissoo Roxb. }\end{array}$ & $\begin{array}{l}\text { Kunch } \\
\text { Not available } \\
\text { Palash } \\
- \\
- \\
\text { Jhun jhuni } \\
- \\
- \\
\text { Bansan, Jhanjhana } \\
\text { Sristi, Rebinea } \\
\text { Chakemdia } \\
\text { - } \\
\text { Sissoo gach }\end{array}$ & $\begin{array}{l}\text { Woody twiner } \\
\text { Woody climber } \\
\text { Tree (medium) } \\
\text { Herb } \\
\text { Herb } \\
\text { Herb } \\
\text { Herb } \\
\text { Herb } \\
\text { Undershrub } \\
\text { Tree } \\
\text { Tree } \\
\text { Tree } \\
\text { Tree }\end{array}$ & $\begin{array}{l}\text { Rare } \\
\text { Rare } \\
\text { Planted } \\
\text { Common } \\
\text { Common } \\
\text { Common } \\
\text { Common } \\
\text { Common } \\
\text { Common } \\
\text { Rare } \\
\text { Rare } \\
\text { Rare } \\
\text { Planted }\end{array}$ \\
\hline
\end{tabular}




\begin{tabular}{|c|c|c|c|c|}
\hline & $\begin{array}{l}\text { D. spinosa Roxb. } \\
\text { D. stipulacea Roxb. } \\
\text { D. volubilis Roxb. } \\
\text { Dendrolobium triangulare (Retz) Merr. } \\
\text { Derris robusta (Roxb. ex DC) Benth. } \\
\text { D. scandens (Roxb.) Benth. } \\
\text { Desmodium monotorium (Houtt.) Merr. } \\
\text { D. triquetrum (L.) DC. } \\
\text { D. gangeticum (L.) DC. } \\
\text { D. heterocarpon (L.) DC. } \\
\text { D. lexiflorum DC. } \\
\text { D. pulchellum (L.) Benth } \\
\text { D. triflorum (L.) DC. } \\
\text { Erythrina indica L. } \\
\text { E. stricta } \text { Roxb. } \\
\text { Flemingia strobilifera (L.) R. Br. } \\
\text { Indigofera tinctoria L. } \\
\text { Mucana pruriens (L.) DC. } \\
\text { M. monosperma DC. } \\
\text { Pongamia pinnata (L.) Pierre } \\
\text { Sesbania grandiflora (L.) Poir. } \\
\text { Tephrosia purpurea (L.) Pers. } \\
\text { T. purpurea (L.) Pers. } \\
\text { T. candida DC. } \\
\text { Uraria rufescens (L.) Desv. Ex DC. } \\
\text { Vicia sativa L. }\end{array}$ & $\begin{array}{l}\text { Ananta, Ananta kantha } \\
\text { Dadbari } \\
\text { Ankilata, Barusirkath } \\
\text { Bir Jarwar } \\
\text { Jangaria, Jamarja, Koroi } \\
\text { Amkurchi } \\
\text { Gorachand, Telegraph plant } \\
\text { - } \\
\text { Salpani } \\
\text { - } \\
\text { - } \\
\text { Jatsalpani } \\
\text { Kulalia, Kodalia } \\
\text { Palita, madar, Mandar } \\
\text { Mandar } \\
\text { Machipatha } \\
\text { Nil } \\
\text { Al-kushi } \\
\text { Soash Guri } \\
\text { Dalkaramcha, Kerum, Pitagoria } \\
\text { Bakful, Bagful, Agati } \\
\text { Bon-nel } \\
\text { Ban-nill } \\
\text { Bilakshani } \\
\text { Dieng-kha-riu } \\
\text { Ankari, Hatka }\end{array}$ & $\begin{array}{l}\text { Shrub (large) } \\
\text { Tree (small) } \\
\text { Woody climber } \\
\text { Shrub } \\
\text { Tree } \\
\text { Woody climber } \\
\text { Undershrub } \\
\text { Undershrub } \\
\text { Undershrub } \\
\text { Undershrub } \\
\text { Undershrub } \\
\text { Shrub } \\
\text { Herb } \\
\text { Tree (medium) } \\
\text { Tree } \\
\text { Shrub } \\
\text { Shrub } \\
\text { Herbaceous climber } \\
\text { Shrubby climber } \\
\text { Tree } \\
\text { Tree (small) } \\
\text { Herb } \\
\text { Herb } \\
\text { Shrub } \\
\text { Shrub } \\
\text { Herb }\end{array}$ & $\begin{array}{l}\text { Common } \\
\text { Rare } \\
\text { Rare } \\
\text { Common } \\
\text { Rare } \\
\text { Common } \\
\text { Common } \\
\text { Common } \\
\text { Common } \\
\text { Common } \\
\text { Common } \\
\text { Common } \\
\text { Common } \\
\text { Planted } \\
\text { Planted } \\
\text { Common } \\
\text { Planted } \\
\text { Common } \\
\text { Common } \\
\text { Planted } \\
\text { Planted } \\
\text { Common } \\
\text { Common } \\
\text { Common } \\
\text { Common } \\
\text { Common }\end{array}$ \\
\hline Fagaceae & $\begin{array}{l}\text { Lithocarpus elegens (Blume) Hatus } \\
\text { Quercus semiserrata Roxb. }\end{array}$ & $\begin{array}{l}\text { Rai batna, Bara cakma } \\
\text { Batna, Guijja batna }\end{array}$ & $\begin{array}{l}\text { Tree } \\
\text { Tree }\end{array}$ & $\begin{array}{l}\text { Rare/Endan } \\
\text { Rare/Endan }\end{array}$ \\
\hline Flacourtiaceae & $\begin{array}{l}\text { Flacourtia jangomas (Lour.) Racusch. } \\
\text { Hydnocarpus kurzii (King) Warb. }\end{array}$ & $\begin{array}{l}\text { Lukluki, Paniala, Paniamala } \\
\text { Chaulmoogra }\end{array}$ & $\begin{array}{l}\text { Tree (medium) } \\
\text { Tree }\end{array}$ & $\begin{array}{l}\text { Rare/Endan } \\
\text { Rare }\end{array}$ \\
\hline Gesneriaceae & Rhynchotoechum ellipticum (Wall. ex Diet.) & - & Undershrub & Common \\
\hline Hydrophyllaceae & Hydrolea zeylanica (L.) Vahl & Kasschara & Herb & Rare \\
\hline Juglandaceae & Engelhardia spicata Lechen ex Blume & Kichra-vhadi, Bolas, Dad & Tree & Rare \\
\hline Lamiaceae & $\begin{array}{l}\text { Anisomeles indica (L.) Kuntze } \\
\text { Hyptis brevipes Poir. } \\
\text { H. suaveolens Poir } \\
\text { Leonurus sibiricus } \text { L. } \\
\text { Leucas aspera } \text { (Willd) Link } \\
\text { L. indica } \text { (L.) R. Br. ex Vatke } \\
\text { L. pulkenetii (Roth.) Spreng. } \\
\text { Ocimum americanum L. } \\
\text { O. basilicum L. } \\
\text { O. sanctum L. } \\
\text { Pogostemon auricularius } \text { (L.) Hassk. }\end{array}$ & $\begin{array}{l}\text { Gubura } \\
- \\
\text { Belatitulshi, Tokma } \\
\text { Roktodron } \\
\text { Choto halkusa } \\
\text { Dondokalosh, Shetodron } \\
\text { Shetodron, Dangalos } \\
\text { Bon-tulshi } \\
- \\
\text { Tulshi } \\
- \\
\end{array}$ & $\begin{array}{l}\text { Herb } \\
\text { Shrub } \\
\text { Herb } \\
\text { Herb } \\
\text { Herb } \\
\text { Herb } \\
\text { Herb } \\
\text { Herb } \\
\text { Herb } \\
\text { Herb } \\
\text { Herb } \\
\end{array}$ & $\begin{array}{l}\text { Common } \\
\text { Common } \\
\text { Common } \\
\text { Rare/Endan } \\
\text { Common } \\
\text { Common } \\
\text { Common } \\
\text { Common } \\
\text { Common } \\
\text { Common } \\
\text { Common } \\
\end{array}$ \\
\hline Lauraceae & $\begin{array}{l}\text { Actinodaphne angustifolia Nees. } \\
\text { Dehaasia kurzii King ex Hook.f. } \\
\text { Litsea glutinosa (Lour.) Robinson } \\
\text { Persea bombycina (King ex Hook.f.) Kosterm. }\end{array}$ & $\begin{array}{l}\text { Tejmatan } \\
\text { Modon Mostho } \\
\text { Kukur-chita, Karjiuki, Menda } \\
\text { Machilus, Nala-amsi }\end{array}$ & $\begin{array}{l}\text { Tree } \\
\text { Tree (medium) } \\
\text { Tree (medium) } \\
\text { Tree (medium) }\end{array}$ & $\begin{array}{l}\text { Common } \\
\text { Rare/Endan } \\
\text { Rare } \\
\text { Rare }\end{array}$ \\
\hline Lecythidaceae & Baringtonia acutangula (L.) Gaertn. & Hijal, Kumia, Hidjal & Tree (medium) & Common \\
\hline Leeaceae & $\begin{array}{l}\text { Leea acuminata Wall. } \\
\text { L. aequata } \mathrm{L} . \\
\text { L. crispa } \mathrm{L} . \\
\text { L. indica (Burm. F.) Merr. } \\
\text { L. macrophylla }\end{array}$ & $\begin{array}{l}\text { Phupharia } \\
\text { Kakjangha } \\
\text { Banchalita, Mach } \\
\text { Kurkur jihwa }\end{array}$ & $\begin{array}{l}\text { Shrub } \\
\text { Shrub } \\
\text { Shrub } \\
\text { Large shrub/ Small tree } \\
\text { Tree (small) } \\
\end{array}$ & $\begin{array}{l}\text { Rare } \\
\text { Rare } \\
\text { Rare } \\
\text { Common } \\
\text { Rare } \\
\end{array}$ \\
\hline Loganiaceae & Fagraea ceilanica Thunb. & - & Tree (small) & Rare \\
\hline
\end{tabular}




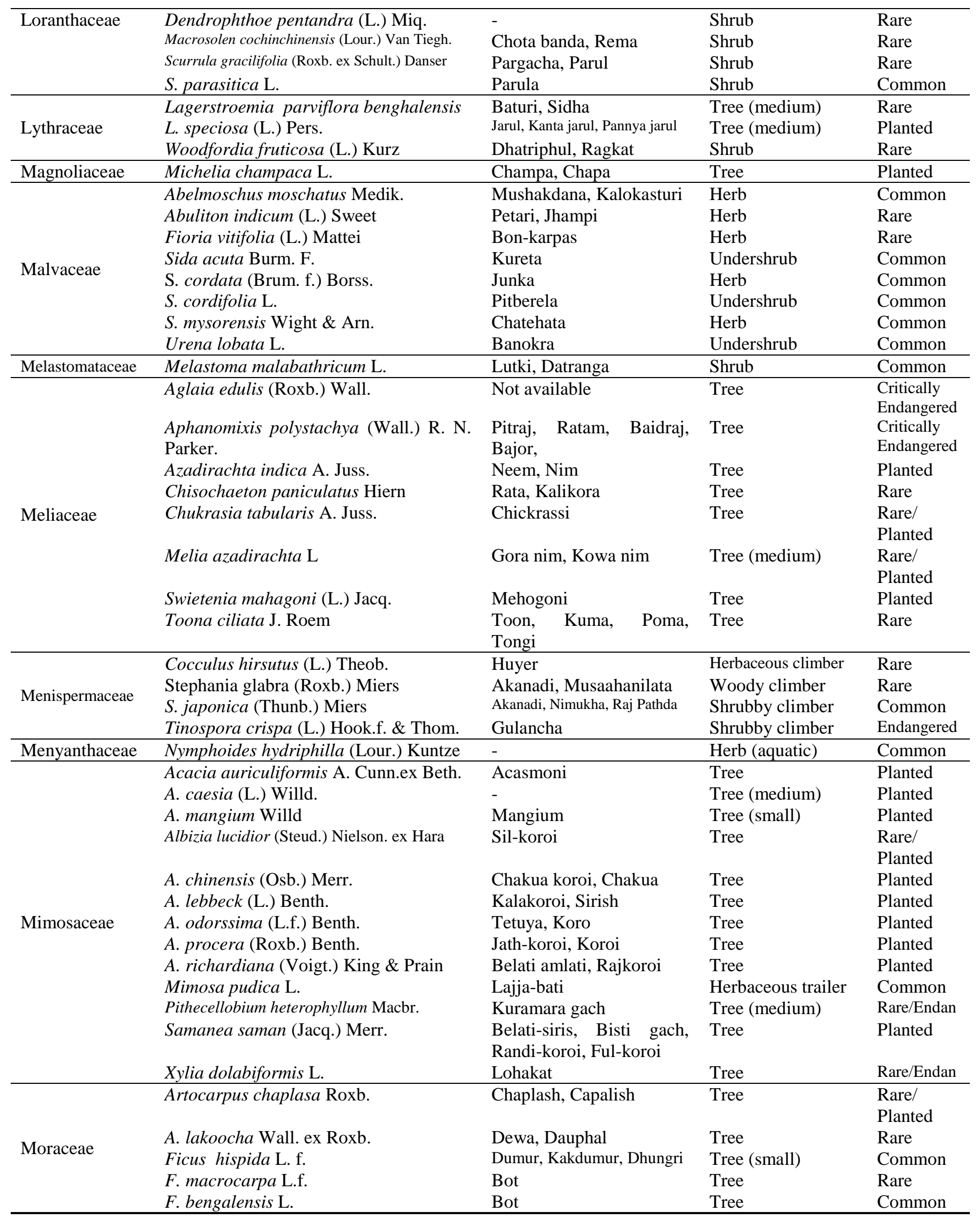




\begin{tabular}{|c|c|c|c|c|}
\hline & $\begin{array}{l}\text { F. fistulosa Reinwdt. } \\
\text { F. gibbosa } \text { Blume } \\
\text { F. glomerata } 51 \text { ttenuat Roxb. } \\
\text { F. racemosa } \text { L. } \\
\text { F. religiosa } \text { L. } \\
\text { Streblus asper Lour. }\end{array}$ & $\begin{array}{l}- \\
\text { Bot } \\
\text { Bot } \\
\text { Jog-dumur } \\
\text { Ashwatha, Ashwath } \\
\text { Sheora, Arga }\end{array}$ & $\begin{array}{l}\text { Tree (small) } \\
\text { Tree } \\
\text { Tree (small) } \\
\text { Tree } \\
\text { Tree } \\
\text { Tree }\end{array}$ & $\begin{array}{l}\text { Common } \\
\text { Common } \\
\text { Common } \\
\text { Common } \\
\text { Common } \\
\text { Common } \\
\end{array}$ \\
\hline Myristicaceae & $\begin{array}{l}\text { Knema erratica (Hook.f. \& Thom.) J. } \\
\text { Sinclair }\end{array}$ & Not available & Tree & $\begin{array}{l}\text { Critically } \\
\text { Endangered }\end{array}$ \\
\hline Myrsinaceae & $\begin{array}{l}\text { Ardisia colorata Roxb. } \\
\text { A. paniculata Roxb. } \\
\text { A. solanacea } \text { Roxb. } \\
\text { Maesa attenua A. DC. } \\
\text { M. indica (Roxb.) DC. } \\
\text { M. paniculata A. DC. } \\
\text { M. ramantacea Wall. }\end{array}$ & $\begin{array}{l}- \\
- \\
\text { Ban-jam } \\
\text { Naricha gach } \\
\text { Sesu, Sirkhi } \\
\text { Noa-maricha } \\
\text { Noa-maricha }\end{array}$ & $\begin{array}{l}\text { Tree (small) } \\
\text { Tree (small) } \\
\text { Shrub } \\
\text { Large shrub/Small tree } \\
\text { Large shrub/Small tree } \\
\text { Tree (small) } \\
\text { Large shrub/Small tree }\end{array}$ & $\begin{array}{l}\text { Rare/Endan } \\
\text { Rare/Endan } \\
\text { Rare/Endan } \\
\text { Common } \\
\text { Common } \\
\text { Common } \\
\text { Common }\end{array}$ \\
\hline Myrtaceae & $\begin{array}{l}\text { Cleistocalyx nervosum paniala } \\
\text { Euclalyptus citrodora Hook. } \\
\text { Syzygium cumini (L.) Skeets } \\
\text { S. cymosum DC. } \\
\text { S. formosum (Wall.) Masamune. } \\
\text { S. fruticosum DC. } \\
\text { S. macrocarpa (Roxb.) Balak. } \\
\text { S. praecox (Roxb.) Rathakr \& N.C. Nair } \\
\text { S. aqueum (Burm. F.) Alston } \\
\text { S. Jambos (L.) Alston }\end{array}$ & $\begin{array}{l}\text { Bhutijam, Godajam } \\
\text { Eucalyptus } \\
\text { Jam, Kalajam } \\
\text { Khudijam, Khurijam, Jonkijam } \\
\text { Paniya jam, Phul jam } \\
\text { Banjam, Putijam, Khudijam } \\
\text { Banjam, Chalta-jam } \\
\text { Dholi jam, Poorajam } \\
\text { Jambo } \\
\text { Golapjam }\end{array}$ & $\begin{array}{l}\text { Tree } \\
\text { Tree } \\
\text { Tree } \\
\text { Tree } \\
\text { Tree } \\
\text { Tree (small) } \\
\text { Tree (medium) } \\
\text { Tree (small) } \\
\text { Tree (medium) } \\
\text { Tree (medium) }\end{array}$ & $\begin{array}{l}\text { Rare } \\
\text { Planted } \\
\text { Planted } \\
\text { Planted } \\
\text { Planted } \\
\text { Rare } \\
\text { Planted } \\
\text { Planted } \\
\text { Planted } \\
\text { Planted }\end{array}$ \\
\hline Nympheaceae & Nymphaea nouchali Brum. F. & Sada Sapla & Herb (Aquatic) & Common \\
\hline Ochnaceae & Ochna wallichii Planch. & Champabaha & Tree (small) & Rare/Endan \\
\hline Oleaceae & $\begin{array}{l}\text { Jasminum sambac (L.) Aiton } \\
\text { J. scandens Vahl } \\
\text { Myxopyrum smilacifolium (Wall.) Blume }\end{array}$ & $\begin{array}{l}\text { Belphul, Beli } \\
-- \\
--\end{array}$ & $\begin{array}{l}\text { Shrubby climber } \\
\text { Woody climber } \\
\text { Scandent Shrub }\end{array}$ & $\begin{array}{l}\text { Rare } \\
\text { Rare } \\
\text { Rare/Endan }\end{array}$ \\
\hline Onagraceae & $\begin{array}{l}\text { Ludwigia hyssopifolia (G. Don.) Exell. } \\
\text { L. adscendens (L.) Hara }\end{array}$ & Keshardam & $\begin{array}{l}\text { Herb } \\
\text { Herb }\end{array}$ & $\begin{array}{l}\text { Common } \\
\text { Common }\end{array}$ \\
\hline Orobanchaceae & Aeginetia indica $\mathrm{L}$. & - & Herb & Common \\
\hline Oxalidaceae & Oxalis corniculata $\mathrm{L}$. & Amrul & Herb & Common \\
\hline Passifloraceae & $\begin{array}{l}\text { Adenia trilobata (Roxb.) Eng. } \\
\text { Passiflora foetida L. }\end{array}$ & $\begin{array}{l}\text { Akandaphal } \\
\text { Jomkalata }\end{array}$ & $\begin{array}{l}\text { Herbaceous climber } \\
\text { Herb }\end{array}$ & $\begin{array}{l}\text { Common } \\
\text { Common }\end{array}$ \\
\hline Piperaceae & $\begin{array}{l}\text { Peperomia } 5 \text { lttenuat (L.) Kunth. } \\
\text { Piper longum L. } \\
\text { P. sylvaticum Roxb. }\end{array}$ & $\begin{array}{l}\text { Peperomea } \\
\text { Pipul } \\
\text { Ban Pan }\end{array}$ & $\begin{array}{l}\text { Herb (succulent) } \\
\text { Herb } \\
\text { Herbaceous creeper }\end{array}$ & $\begin{array}{l}\text { Common } \\
\text { Common } \\
\text { Common }\end{array}$ \\
\hline Polygalaceae & $\begin{array}{l}\text { Salomonia ciliata }(\mathrm{L} .) \text { DC. } \\
\text { Xanthophyllum flavescens Roxb. }\end{array}$ & - & $\begin{array}{l}\text { Herb } \\
\text { Tree }\end{array}$ & $\begin{array}{l}\text { Common } \\
\text { Common }\end{array}$ \\
\hline Polygonaceae & $\begin{array}{l}\text { Ampelygonum chinense }(\mathrm{L} .) \text { Lindl. } \\
\text { Persicaria orientalis }(\mathrm{L} .) \text { Spach. } \\
\text { P. hydropiper }(\text { L.) Spach. } \\
\text { P. viscosa Nakia }\end{array}$ & $\begin{array}{l}\text { Mohicharan sak } \\
\text { Bara panimarich } \\
\text { Pakurmul } \\
\text { Atala Biskatali } \\
\end{array}$ & $\begin{array}{l}\text { Herb } \\
\text { Herb } \\
\text { Herb } \\
\text { Herb }\end{array}$ & $\begin{array}{l}\text { Common } \\
\text { Common } \\
\text { Common } \\
\text { Common }\end{array}$ \\
\hline Proteaceae & Helicia excels (Roxb.) Blume & - & Tree (medium) & Rare \\
\hline Rhamnaceae & $\begin{array}{l}\text { Gounia tiliaefolia Lam. } \\
\text { Zizyphus glabrata Heyne ex Roth. } \\
\text { Z. mauritiana Lam. } \\
\text { Z.oenoplia (L.) Mill. }\end{array}$ & $\begin{array}{l}- \\
\text { Pahari boroi } \\
\text { Kul, Boroi } \\
\text { Boot boroi }\end{array}$ & $\begin{array}{l}\text { Scandent Shrub } \\
\text { Shrub } \\
\text { Tree (medium) } \\
\text { Large srub/Small tree }\end{array}$ & $\begin{array}{l}\text { Rare/Endan } \\
\text { Rare } \\
\text { Common } \\
\text { Common }\end{array}$ \\
\hline Rubiaceae & $\begin{array}{l}\text { Aidia oppositifolia (Roxb.) Rahman \& Das } \\
\text { Canthium angustifolium Roxb. } \\
\text { Cerescoides campanulata (Roxb.)Tirveng. } \\
\text { Chasalia curviflora var. ophioxyloodes } \\
\text { Dentella repens (L.) Forst. \& G. Frost. }\end{array}$ & $\begin{array}{l}\text { Bhadi, Dalya phul } \\
\text { Katamalli, Katalichapa } \\
\text { Not available } \\
- \\
\text { Bhuipat }\end{array}$ & $\begin{array}{l}\text { Tree (small) } \\
\text { Shrub } \\
\text { Tree } \\
\text { Shrub } \\
\text { Herb }\end{array}$ & $\begin{array}{l}\text { Rare/Endan } \\
\text { Common } \\
\text { Endangered } \\
\text { Rare } \\
\text { Common }\end{array}$ \\
\hline
\end{tabular}




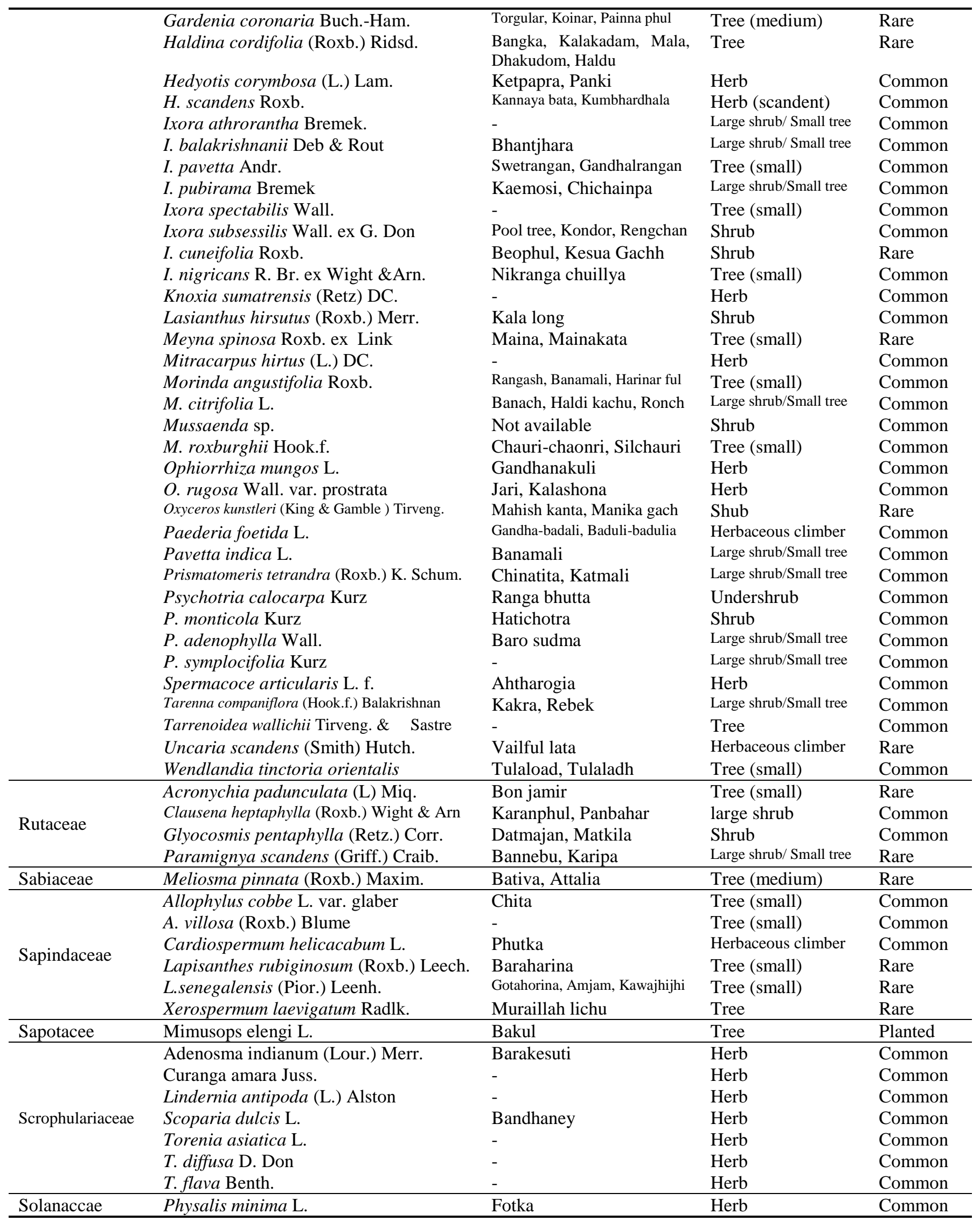




\begin{tabular}{|c|c|c|c|c|}
\hline & $\begin{array}{l}\text { P. micrantha Link. } \\
\text { Solanum nigrum } \mathrm{L} \text {. } \\
\text { S. sisymbrifolium Lam. } \\
\text { S. torvum Sw. } \\
\text { S. virginianum L. }\end{array}$ & $\begin{array}{l}\text { Bantipariya, Phuika, Terare } \\
\text { Gurkhi } \\
\text { Kanta begun } \\
\text { Goth begun } \\
\text { Kantakari } \\
\end{array}$ & $\begin{array}{l}\text { Herb } \\
\text { Herb } \\
\text { Herb } \\
\text { Shrub } \\
\text { Herb } \\
\end{array}$ & $\begin{array}{l}\text { Common } \\
\text { Common } \\
\text { Common } \\
\text { Common } \\
\text { Common } \\
\end{array}$ \\
\hline Sonneratiaceae & Duabunga grandiflora (Roxb. ex DC) Walp & Bandarhulla & Tree & Rare \\
\hline Sphenocleaceae & Sphenoclea zeylanica Gaertn. & Jhil Marich & Herb & Common \\
\hline Sterculiaceae & $\begin{array}{l}\text { Abroma augusta } \text { (L.) L.f. } \\
\text { Byttneria pilosa } \text { Roxb. } \\
\text { Firmiana colorata (Roxb.) R.Br. } \\
\\
\text { Pterospermum semisagittatum Buch.-Ham. } \\
\text { Sterculia foetida } \text { L. } \\
\text { S. hamiltonii (Kuntze) Adelb. } \\
\text { S. villosa Roxb. ex Smith } \\
\text { Styrax serolatus Roxb. }\end{array}$ & $\begin{array}{l}\text { Ulatkambal, Tambol } \\
\text { Harjora-lata,Jumi, Harbanga-lata } \\
\text { Naichicha-udal, Ujal, } \\
\text { Patagota, Samarri, Pisi } \\
\text { Laona assar, Bara assar } \\
\text { Udal } \\
\text {-- } \\
\text { Udal, Chandal } \\
\text { Kanchuni, Fulkat }\end{array}$ & $\begin{array}{l}\text { Large shrub/Small tree } \\
\text { Shrubby climber } \\
\text { Tree } \\
\text { Tree } \\
\text { Tree (medium) } \\
\text { Tree (small) } \\
\text { Tree (medium) } \\
\text { Tree (small) }\end{array}$ & $\begin{array}{l}\text { Common } \\
\text { Common } \\
\text { Critically } \\
\text { Endangered } \\
\text { Rare/Endan } \\
\text { Rare/Endan } \\
\text { Rare/Endan } \\
\text { Rare/Endan } \\
\text { Rare/Endan }\end{array}$ \\
\hline Symplocaceae & Symplocos racemosa Roxb. & - & Tree (medium) & Rare/Endan \\
\hline Theaceae & Eurya attenuate DC. & Lapet, Sagoler bori & Tree (small) & Rare/Endan \\
\hline Tiliaceae & $\begin{array}{l}\text { Corchorus aestuans } \mathrm{L} . \\
\text { Grewia disperma } \text { Roxtt. } \\
\text { G. nervosa (Lour.) Panigr. } \\
\text { Triumfetta pilosa Roth } \\
\text { T. rhomboidea Jacq. }\end{array}$ & $\begin{array}{l}\text { Mass, Masjot, Moos } \\
\text { Titapat } \\
\text { Laona assar, Dhaman } \\
\text { Assar, Pisla, Patka } \\
- \\
\text { Ban-okra } \\
\end{array}$ & $\begin{array}{l}\text { Herb } \\
\text { Tree (medium) } \\
\text { Tree (small) } \\
\text { Herb } \\
\text { Herb } \\
\end{array}$ & $\begin{array}{l}\text { Critically } \\
\text { Endangered } \\
\text { Rare } \\
\text { Common } \\
\text { Common } \\
\text { Common } \\
\text { Common } \\
\end{array}$ \\
\hline Ulmaceae & Trema orientalis (L.) Blume & Bonjiyal, Jiban, Dhalagar, Dhola & Tree (small) & Common \\
\hline Urticaceae & $\begin{array}{l}\text { Boehmeria glomerulifera Miq. } \\
\text { Pouzolzia zeylanica (L.) Benn. } \\
\text { Sarcochlamys pulcherrima Gaud. }\end{array}$ & $\begin{array}{l}\text { Borthurthuri } \\
\text { Kullaruki } \\
\text { Acila }\end{array}$ & $\begin{array}{l}\text { Tree (small) } \\
\text { Herb } \\
\text { Large shrub }\end{array}$ & $\begin{array}{l}\text { Common } \\
\text { Common } \\
\text { Common } \\
\end{array}$ \\
\hline Verbenaceae & $\begin{array}{l}\text { Callicarpa arborea } \text { Roxb. } \\
\text { Clerodendrum indicum (L.) Kuntze } \\
\text { C. viscosum Vent. } \\
\text { C. wallichii Merr. } \\
\text { Duranta erecta } \text { L. } \\
\text { Gmelina arborea } \text { Roxb. } \\
\text { Lantana camara } \text { L. } \\
\text { Lippia alba } \text { Mill. N. E. Br. ex Brit. \& Wilson } \\
\text { Phyla nodiflora } \text { (L.) Greene } \\
\text { Premna esculenta } \text { Roxb. } \\
\text { Tectona grandis } \text { L. f. } \\
\text { Vitex glabrata } \text { R. Br. } \\
\text { V. negundo } \text { L. } \\
\text { V. peduncularis Wall. ex Schauer } \\
\text { V. trifolia } \text { L. }\end{array}$ & $\begin{array}{l}\text { Barmala } \\
\text { Bamunhati } \\
\text { Bhant, Ghetu phul } \\
\text { Malbong, Dieng-julkah } \\
\text { Durantha, Katamehedi } \\
\text { Gamari } \\
\text { Lantana } \\
\text { Pichas-lakr } \\
\text { Bhuiokra, Karoghar } \\
\text { Lalana, Lalong } \\
\text { Shegun, Shegoon, Teak } \\
\text { Goda, Horina, Aswal } \\
\text { Baranishinda, Nishinda } \\
\text { Goda, Horina, Arswal } \\
\text { Choto nishinda }\end{array}$ & $\begin{array}{l}\text { Tree (medium) } \\
\text { Shrub } \\
\text { Shrub } \\
\text { Shrub } \\
\text { Large shrub } \\
\text { Tree } \\
\text { Shrub } \\
\text { Shrub } \\
\text { Herb } \\
\text { Shrub } \\
\text { Tree } \\
\text { Tree } \\
\text { Tree (small) } \\
\text { Tree } \\
\text { Shrub }\end{array}$ & $\begin{array}{l}\text { Common } \\
\text { Common } \\
\text { Common } \\
\text { Rare } \\
\text { Common } \\
\text { Planted } \\
\text { Common } \\
\text { Common } \\
\text { Common } \\
\text { Common } \\
\text { Planted } \\
\text { Rare } \\
\text { Common } \\
\text { Rare } \\
\text { Common }\end{array}$ \\
\hline Vitaceae & $\begin{array}{l}\text { Ampelocissus barbata (Wall.) Planch. } \\
\text { Cayratia japonica (Thunb.) Gagnep. } \\
\text { C. trifolia (L.) Domin } \\
\text { Cissus assamica (Laws.) Craib } \\
\text { C. elongata } \text { Roxb. } \\
\text { C. javana DC. } \\
\text { C. pentagona Roxb. } \\
\text { Tetrastigma angustifolia (Roxb.) Deb } \\
\text { T. bracteolatum (Roxb.) Planch. } \\
\text { T. leucostaphylum (Dennst.) Alston }\end{array}$ & $\begin{array}{l}\text { Jarila-lahari } \\
- \\
\text { Amallat, Amal lata } \\
\text { Amasha lata } \\
\text { Dhemna, Chemna } \\
\text { Bichitra lata } \\
\text { Sona-lota, Sona-tola } \\
\text { Nekung riubi } \\
\text { Golgoti lata } \\
\text { Horinia-lata, Jarul lata }\end{array}$ & $\begin{array}{l}\text { Herbaceous climber } \\
\text { Herbaceous climber } \\
\text { Herbaceous climber } \\
\text { Herbaceous climber } \\
\text { Herbaceous climber } \\
\text { Herbaceous climber } \\
\text { Herbaceous climber } \\
\text { Herbaceous climber } \\
\text { Herbaceous climber } \\
\text { Woody climber }\end{array}$ & $\begin{array}{l}\text { Common } \\
\text { Common } \\
\text { Common } \\
\text { Rare } \\
\text { Common } \\
\text { Rare } \\
\text { Rare } \\
\text { Rare } \\
\text { Common } \\
\text { Common }\end{array}$ \\
\hline
\end{tabular}


It revealed from Table 2 that 22 threatened plants, reported in the Red Data Book of Bangladesh (Khan et al. 2001, Rahman 2013 and Ara et al. 2013), were located to the area of Hazarikhil WS. These threatened species in this Wildlife Sanctuary are likely to be under conservation management.

The study also resulted with the rediscovery of 3 species which were thought to be possibly extinct from the flora of Bangladesh since these have neither been collected nor reported to occur elsewhere in Bangladesh for more than 100 years after their first record (Rahman 2013) and these are:

Aglaia edulis (Roxb.) Wall. (Meliaceae)

It was recorded from Sylhet by J. D. Hooker in 1875 (Hook.f. 1875). Since then no report of its occurrence in Bangladesh was available. It is being rediscovered from Hazarikhil WS.

Knema erratica (Hook.f. \& Thom.) J. Sinclair (Myristicaceae)

Basionym: Myristica erretica Hook.f. and Thom. (1855). It was first recorded from Chittagong by Hook.f. \& Thom. and thereafter by Prain (1903) as Myristica longifolia Wall. var. erratica (Hook.f. and Thom.) Hook.f. (1885). Since then no report of its collection from elsewhere in Bangladesh was available. The present collection was made from Hazarikhil WS after more than 100 years.

Artabotrys caudatus Wall ex Hook.f. and Thom. (Annonaceae)

This species was recorded from Sylhet by J. D. Hooker and T. Thomson in 1855. Since then it has neither been collected nor reported to occur elsewhere in Bangladesh, and hence reported as possibly extinct (Rahman 2013). It is being rediscovered from Hazarikhil WS.

Table 2. Some rare and endangered species of Bangladesh relocated to Hazarikhil WS (as per RDB).

\begin{tabular}{|c|c|c|c|c|}
\hline Family & Threatened species & Bangla name & Habit & $\begin{array}{l}\text { Status of } \\
\text { occurrence* }\end{array}$ \\
\hline \multirow{3}{*}{ Apocynaceae } & Alstonia nerifolia D. Don & Chatim & Tree & EN \\
\hline & Melodinus monogynus Roxb. & - & Woody twiner & $\mathrm{EN}$ \\
\hline & Wrightia arborea (Dennst.) Mabb. & Dudh-koraiya & Tree & EN \\
\hline Myrsinaceae & Ardisia colorata Roxb. & -- & Small tree & VU \\
\hline \multirow{3}{*}{ Anacardiaceae } & Buchanania lancifolia Roxb. & Chikki & Tree & NE \\
\hline & Mangifera sylvatica Roxb. ex Wall. & Uri Aam, Jangali Aam, Laksmi Aam & Tree & NE \\
\hline & Swintonia floribunda Griff. & Civit, Chundul, Am chundul & Tree & DD \\
\hline Tiliaceae & Brownlowia elata Roxb. & Mass, Masjot, Moos & Tree & VU \\
\hline Lauraceae & Dehaasia kurzii King ex Hook.f. & Modon Mostho & Medium tree & VU \\
\hline \multirow[t]{2}{*}{ Rubiaceae } & Wendlandia tinctoria orientalis & Tulaload, Tulaladh & Small tree & EN \\
\hline & Psychotria symplocifolia Kurz & -- & Large shrub & EN \\
\hline Sterculiaceae & Pterospermum semisagittatum Buch.-Ham. & Laona assar, Bara assar & Tree & DD \\
\hline \multirow{2}{*}{ Menispermaceae } & Stephania glabra (Roxb.) Miers & Akanadi, Musaahanilata & Woody climber & VU \\
\hline & Tinospora crispa (L.) Hook.f. \& Thom. & Gulancha & Shrubby climber & EN \\
\hline Sapindaceae & Xerospermum laevigatum Radlk. & Muraillah lichu & Tree & VU \\
\hline
\end{tabular}

*EN=Endangered, VU=Vulnerable, DD=Data deficient, NE=Near threatened 
This rediscovery indicates that many species which are thought to be possibly extinct from Bangladesh still could be existed in the biodiversity reserves of Chittagong and Hill Tracts. Conservation of a Protected Area for its floristic elements, wild lives, ecosystem and habitats depend largely on its sustainable Management Plan and proper implementation. Conservation management of this WS, as assessed, is seemed to be poor for which natural habitats are being degraded and floristic elements depleting at an alarming rate although the creation of planted forests is increased. Both core and buffer zones of fragmented reserves are largely affected by illegal encroachments, manmade forest fire, hunting and over exploitation of rare-endangered species and lack of alternative livelihood management for forest inhabitants etc.

Based on present survey, a total of 478 species belonging to 88 dicot families are recognized to be represented in the flora of Hazarikhil WS. Of these, about 39.54\% species are trees; $18 \%$ species are rare and endangered (EN) and $2.93 \%$ species are critically endangered (CR). The floristic diversity of this sanctuary is comparatively richer than Rampahar, Sitapahar and Chunati wildlife sanctuaries in terms of total land area.

The floristic and faunal elements of this Wildlife Sanctuary are the dependable source of food, medicine, water, timber and fuel wood of the local inhabitants. The floral biodiversity of the sanctuary exists under different degraded ecosystems and its protection management is still inadequate.

Sustainable Management Plan of the Protected Area in the light of National Conservation Strategy is to be made and proper implementation through action plan is urgently required with collaboration and cooperation of stakeholders and local administrations. Specially rare and endangered species along with their degraded habitats, as recognized in this study, are suggested to protect taking proper conservation management.

\section{ACKNOWLEDGEMENTS}

The study has been carried out to support with floristic data for the preparation of a Protected Area Management Plan for Hazarikhil WS under Strengthening Regional Co-operation for Wildlife Protection Project (SRCWP) for which author is thankful to the Forest Department, GoB.

\section{REFERENCES}

Ahmed, Z. U., M. A. Hassan, Z. N. T. Begum, M. Khondker, S. M. H. Kabir, M. Ahmad and A. T. A. Ahmed (eds.). 2007, 2008, 2009. Encyclopedia of Flora and Fauna of Bangladesh. Vols. 6-12.

Ara, H., B. Khan and S. N. Uddin. 2013. Data Book of Vascular Plants of Bangladesh. Vol. 2. Bangladesh National Herbarium, Dhaka, pp. 1-280.

Das, D. K. and M. K. Alam. 2001. Trees of Bangladesh. BFRI, Chittagong, pp. 1-342.

Dey, T. K. 1995. Useful Plants of Bangladesh. Chittagong, pp. 1-1055.

Dey, C. K., M. A. Rahman and C. C. Wilcock. 1999. An enumeration of Tree species of Chittagong. Biod. Bull. Bangladesh. 1: 1- 81.

Heinig, R. L. 1925. List of Plants of Chittagong Collectorate and Hill Tracts. Darjeeling, India, pp.1-84.

Hooker, J. D. 1872-1897. The Flora of British India. Vols. 1-7. Bishen Singh Mahendra Pal Singh, Dehr Dun, India.

Huq, A. M. 1986. Plant Names of Bangladesh. Bangladesh National Herbarium, Dhaka. 289 pp.

Khan, M. S. 1977. Flora of Bangladesh. Vol. 4. Bangladesh National Herbarium, Dhaka. 
Khan, M. S., M. M. Rahman and M. A. Ali. (eds). 2001. Red Data Book of Vascular Plants of Bangladesh. Bangladesh National Herbarium, Dhaka, pp. 1-179.

Prain, D. 1903 (reprint 1963). Bengal Plants. Vol. I-II. Botanical Survey of India, Bishen Singh Mahendra Pal Singh, Dehra Dun, India, pp. 1-1013.

Rahman, M. A. (ed). 2013. IUCN Red List Categories of Plants: Red Data Book of Flowering Plants of Bangladesh. Vol. 1. Published by Prof. Dr. M. Atiqur Rahman, Chittagong, pp. 1-256.

Rashid, M. E. and M. A. Rahman. 2011. Updated Nomenclature and Taxonomic Status of the Plants of Bangladesh included in Hook.f., The Flora of British India: Volume I. Bangladesh J. Plant Taxon.18(2): 177-197.

Rashid, M. E. and M. A. Rahman. 2012. Updated nomenclature and taxonomic status of the plants of Bangladesh included in Hook.f., The Flora of British India: Volume II. Bangladesh J. Plant Taxon. 19(2): 173-190. 